\title{
MiR-203 acts as a radiosensitizer of gastric cancer cells by directly targeting ZEBI
}

This article was published in the following Dove Press journal: OncoTargets and Therapy

\author{
Ying Jiang \\ Shan Jin \\ Shisheng Tan \\ Qi Shen \\ Yingbo Xue
}

Department of Oncology, Guizhou Provincial People's Hospital, Guiyang, Guizhou, People's Republic of China
Correspondence: Yingbo Xue Department of Oncology, Guizhou Provincial People's Hospital, No. 83, East Zhongshan Road, Guiyang City 550002, Guizhou Province, People's Republic of China

Tel +86085I85605389

Email Imdrz76@I26.com
Objective: Gastric cancer (GC) is a common tumor malignancy with high incidence and poor prognosis. Radiotherapy is one of the main strategies for GC treatment, while development of radioresistance limits the effectiveness. microRNA-203 (miR-203) has been reported to participate in progression of $\mathrm{GC}$, whereas its interaction with radiosensitivity of GC and the related mechanism remain largely unclear.

Methods: The expressions of miR-203 and zinc finger E-box binding homeobox 1 (ZEB1) were measured in GC tissues and cells by quantitative real-time polymerase chain reaction or western blot. Survival fraction, cell viability and apoptosis were measured in GC cells after treatment of radiation by colony formation, 3-(4,5-dimethyl-2-thiazolyl)-2,5-diphenyl-2-Htetrazolium bromide (MTT) assay or flow cytometry, respectively. Tumor volume and weight were detected in murine xenograft model after radiation treatment. The interaction between miR-203 and ZEB1 was explored by bioinformatics analysis and luciferase activity assay.

Results: miR-203 expression was down-regulated and ZEB1 mRNA level was up-regulated in GC. The expression of miR-203 was associated with radiosensitivity of GC cells. Moreover, overexpression of miR-203 decreased survival fraction, cell viability and tumor growth but promoted cell apoptosis in radiation-treated GC cells. However, knockdown of miR-203 played an opposite effect. ZEB1 was validated as a target of miR-203, and it was involved in miR-203-mediated radiosensitivity of GC cells in vitro and in vivo.

Conclusion: miR-203 promoted radiosensitivity of GC cells by targeting ZEB1, indicating miR-203 as a promising radiosensitizer for GC treatment.

Keywords: gastric cancer, miR-203, ZEB1, radiosensitizer, radiosensitivity

\section{Introduction}

Gastric cancer (GC) is one of the most common tumor malignancies with high mortality and incidence worldwide. ${ }^{1}$ With the advance of understanding the pathogenesis, many strategies have been exploited in the diagnosis and management of GC, such as surgical resection, chemotherapy and radiotherapy. ${ }^{2}$ Radiotherapy is one of the main modalities of cancer treatment and plays important roles in treatment of gastrointestinal cancers, such as cancers of the stomach, pancreas, esophagus, liver and anus. ${ }^{3}$ Moreover, the progress of preoperative and postoperative radiotherapy has gained more attention in GC. ${ }^{4}$ However, development of radioresistance induces the failure of radiation treatment. Hence, it is urgent to explore novel radiosensitizers to improve the effectiveness of radiotherapy.

microRNAs (miRNAs) are a class of small noncoding RNAs, which have been indicated as potential targets for improving radiotherapy. ${ }^{5}$ Moreover, increasing works have demonstrated that miRNAs play essential roles in diagnosis, prognosis and 
radiotherapy in GC. ${ }^{6,7}$ For example, miR-196b enhances the radiosensitivity of $\mathrm{GC}$ cells by targeting radiation sensitive protein 23 homologue B (RAD23B). ${ }^{8}$ Former works suggest that miR-203 has a vital role in proliferation, metastasis and apoptosis in ovarian cancer, hepatocellular carcinoma and prostate cancer. ${ }^{9-11}$ In addition, miR-203 contributes to radiosensitivity by inhibiting DNA damage repair via regulating phosphatidylinositol 3-kinase (PI3K)/protein kinase B (AKT) and Janus kinase (JAK)/signal transducer and activators of transcription 3 (STAT3) pathways in glioma cells. ${ }^{12}$ This reveals that miR-203 may serve as a radiosensitizer for cancer therapy. Increasing efforts have reported that miR-203 inhibits invasion, metastasis and growth by regulating multiple pathways in GC. ${ }^{13-15}$ However, little is known about the role and mechanism of miR-203 in radiosensitivity of GC cells.

Zinc finger E-box binding homeobox 1 (ZEB1) is a key element controlling epithelial-to-mesenchymal transition, which is implicated in tumorigenesis and development of human cancers. ${ }^{16}$ Furthermore, ZEB1 is suggested to have an impact on the outcome of therapy resistance, including radioresistance and drug resistance. ${ }^{17}$ Notably, ZEB1 is indicated as an oncogene to promote cell viability, migration and invasion in GC. ${ }^{18}$ Besides, available evidence indicates the regulatory network of miR-203/ZEB1 in progression of non-small cell lung cancer. ${ }^{19}$ Thus, we hypothesized that ZEB1 might be required for miR-203mediated regulation of radiosensitivity in GC. In this study, we first analyzed the effect of miR-203 on radiosensitivity of GC in vitro and in vivo and explored the potential interaction between miR-203 and ZEB1.

\section{Materials and methods}

\section{Tissues samples}

A total of 36 paired tumor and adjacent normal tissues (NT) were collected from patients with GC in Guizhou Provincial People's Hospital and then immediately stored at $-80^{\circ} \mathrm{C}$ until RNA extraction. All patients enrolled in this study had no history of chemotherapy or radiotherapy prior to surgery and signed the informed consent. This study was approved by the Research Ethics Committee of Guizhou Provincial People's Hospital and performed in accordance with the Declaration of Helsinki.

\section{Cell culture and transfection}

The human normal gastric epithelium cell line (GES-1) and GC cell lines (MKN-45, SGC-7901 and AGS) cells were purchased from American Tissue Culture Collection (Manassas, VA, USA) and cultured in Dulbecco's Modified Eagle Medium (Gibco, Carlsbad, CA, USA) containing 10\% fetal bovine serum (Gibco), $100 \mathrm{U} / \mathrm{mL}$ penicillin and $100 \mu \mathrm{g} /$ $\mathrm{mL}$ streptomycin (Invitrogen, Carlsbad, CA, USA) at $37^{\circ} \mathrm{C}$ in a humidified atmosphere with $5 \% \mathrm{CO}_{2}$.

miR-203 mimic (miR-203), miRNA negative control (NC) (miR-NC), miR-203 inhibitor (anti-miR-203), inhibitor NC (anti-miR-NC), ZEB1 overexpression vector (ZEB1), pcDNA empty vector (vector), short hairpin RNA (shRNA) against ZEB1 (shZEB1) and shRNA NC (scrambled) were synthesized by Genepharma (Shanghai, China). Cell transfection was performed in MKN-45 and AGS cells for $48 \mathrm{hrs}$ by using Lipofectamine 2000 (Invitrogen) following the manufacturer's instructions. The non-transfected cells were regarded as blank group.

\section{Colony formation}

MKN-45 and AGS cells were exposed with different doses of radiation $(0,2,4,6 \text { and } 8 \mathrm{~Gy})^{8}$ using the X-ray apparatus (Rad Source Technologies, Alpharetta, GA, USA) with a dose rate of $200 \mathrm{cGy} / \mathrm{min}$. Every sample was prepared in triplicate. After radiation for $24 \mathrm{hrs}$, cells were collected and seeded into 12-well plates at a density of 300 cells/well. After culture for $10 \mathrm{~d}$, treated cells were fixed with methanol (Sigma, St. Louis, MO, USA) for $30 \mathrm{mins}$ and then stained with $0.01 \%$ crystal violet (Sigma) for 15 mins. The colony formation was analyzed under a microscope (Olympus, Tokyo, Japan) and survival fraction was calculated as number of colonies/number of cells plated by normalizing to the control cells (non-irradiation).

\section{Quantitative real-time polymerase chain reaction}

Total RNA was isolated from tissues or cells by using TRIzol reagent (Invitrogen) according to the manufacturer's instructions. The RNA was reversely transcribed using TaqMan microRNA Reverse Transcription Kit (Applied Biosystems, Foster City, CA, USA) or M-MLV Reverse Transcription Kit (Thermo Fisher, Wilmington, DE, USA). The complementary DNA (cDNA) was used for quantitative real-time polymerase chain reaction using SYBR green (Applied Biosystems) following the manufacturer's instructions. Every sample was prepared in triplicate, and the experiment was repeated three times. The expressions of miR-203 and ZEB1 were calculated using $2^{-\Delta \Delta \mathrm{Ct}}$ method with U6 small RNA or GAPDH as internal 
control, respectively. ${ }^{20}$ The primers were list as follows: miR-203 (Forward, 5'-ACACTCCAGCTGGGGTGAAAT GTTTA-3'; Reverse, 5'-TGGTGTCGTGGAGTCG-3'), U6 (Forward, 5'-GCTTCGGCAGCACATATACTAAAAT-3'; Reverse, 5'-CGCTTCACGAATTTGCGTGTCAT-3'), ZEB1 (Forward, 5'-AGCGAGGTAAAGTTGCGTCT-3'; Reverse, 5'-AGGTTTTCTGGGCCATACCG-3'), GAPDH (Forward, 5'-AACGGATTTGGTCGTATTGGG-3'; Reverse， 5'-TCG CTCCTGGAAGATGGTGAT-3').

\section{Cell viability}

3-(4,5-dimethyl-2-thiazolyl)-2,5-diphenyl-2-H-tetrazolium bromide (MTT) assay was conducted to measure cell viability. MKN-45 and AGS cells were exposed with different doses of radiation (0, 2, 4, 6 and 8 Gy). Treated cells were seeded into 96 -well plates at a density of $1 \times 10^{4}$ per well and each group was prepared in triplicate. After the culture for $48 \mathrm{hrs}$, cells were interacted with $5 \mathrm{mg} / \mathrm{mL}$ MTT $(10 \mu \mathrm{l})$ solution (Thermo Fisher) for another $4 \mathrm{hrs}$. Subsequently, $100 \mu \mathrm{L}$ of dimethylsulfoxide (DMSO, Thermo Fisher) was added and incubated for 10 mins. The absorbance was measured at $490 \mathrm{~nm}$ using a microplate reader (Bio-Rad, Hercules, CA, USA).

\section{Cell apoptosis}

Cell apoptosis was detected using Annexin V-fluorescein isothiocyanate (FITC)/propidium iodide (PI) apoptosis detection kit (Sigma) via flow cytometry. MKN-45 and AGS cells $\left(2 \times 10^{5}\right)$ were seeded into 6-well plates, treated with radiation of $6 \mathrm{~Gy}^{21}$ and cultured for $48 \mathrm{hrs}$. Each group was prepared in triplicate. Subsequently, cells were washed with PBS and resuspended in binding buffer, followed by staining with $10 \mu \mathrm{L}$ Annexin V-FITC for 10 mins and $5 \mu \mathrm{L}$ PI for 10 mins in the dark according to the manufacturer's instructions. The apoptotic (FITC positive and PI positive or negative) cells were analyzed by using a flow cytometer (Becton Dickinson, Franklin Lakes, NJ, USA).

\section{Tumor radiosensitivity assay in vivo}

BALB/c nude mice (male, 4-week-old) were obtained from Vital River Laboratory Animal Technology (Beijing, China) and housed in specific pathogen-free conditions with a 12-hr light/dark cycle and free access to water and food for one week. Every experiment was made to minimize animals ( $\mathrm{n}=7$ per group) under the approval of the Animal Research Committee of Guizhou Provincial People's Hospital and conducted in conformity with the guidelines of Care and Use of Laboratory Animal. MKN45 cells were transfected with the lentiviral vectors with miR-NC, miR-203, miR-203+Vector or miR-203+ ZEB1 constructed by GeneCopoeia (Rockville, MD, USA). AGS cells were transfected with the lentiviral vectors with antimiR-NC, anti-miR-203, anti-miR-203+scrambled or antimiR-203+ shZEB1. Stably transfected cells $\left(5 \times 10^{6}\right)$ were subcutaneously injected into the left flank of mice. After 1 week following the inoculation, mice were treated with radiation of $5 \mathrm{~Gy}^{22}$ and then tumor volumes were measured every 3 days with the formula: volume $\left(\mathrm{mm}^{3}\right)=$ width $^{2} \times$ length $/ 2$. On $28 \mathrm{~d}$ after injection, the mice were sacrificed and tumor samples were weighted.

\section{Luciferase activity assay}

The potential targets of miR-203 were predicted by bioinformatics analysis using TargetScan Release 7.2 (http:// www.targetscan.org/vert_72/). The 3' untranslated regions (3'-UTR) sequences of ZEB1 containing binding sites of miR-203 were amplified and then cloned into the firefly luciferase-expressing pGL3-Promoter vectors (Promega, Madison, WI, USA) to synthesize wild-type (Wt) luciferase reporter vectors (ZEB1-Wt). MKN-45 and AGS cells were co-transfected with $20 \mathrm{ng}$ ZEB1-Wt or empty vector, $15 \mathrm{ng}$ renilla luciferase vector (control vector) and $40 \mathrm{nM}$ miR-203, miR-NC, anti-miR-203 or anti-miR-NC using Lipofectamine 2000 according to the manufacturer's protocols. After the transfection for $48 \mathrm{hrs,} \mathrm{luciferase}$ activity assay was analyzed with luciferase assay kit (Promega) according to the manufacturer's instructions.

\section{Western blot}

Proteins were isolated from cells by using RIPA lysis buffer (Beyotime Biotechnology, Shanghai, China) and quantified by BCA protein assay kit (Beyotime Biotechnology). After the denaturation, equal amounts of proteins were separated by SDS-PAGE gel electrophoresis and transferred to polyvinylidene difluoride (PVDF) membranes (Millipore, Billerica, MA, USA). The membranes were blocked with $5 \%$ non-fat milk for $1 \mathrm{hr}$ at room temperature, and then incubated with primary antibodies against ZEB1 (ab203829, 1:500 dilution, Abcam, Cambridge, UK) or GAPDH (ab181602, 1:10,000 dilution, Abcam) overnight at $4^{\circ} \mathrm{C}$ and horseradish peroxidase (HRP)-conjugated secondary antibody (ab205718, 1:10,000 dilution, Abcam) for $2 \mathrm{hrs}$ at room temperature. GAPDH was regarded as loading control in this study. The protein blots were visualized 
using enhanced chemiluminescence chromogenic substrate (Beyotime Biotechnology).

\section{Statistical analysis}

Three independent experiments were conducted for all analyses and the data were presented as the mean \pm standard deviation (SD). The relationship between the expression of miR-203 and ZEB1 was measured by Spearman rank correlation. Student's $t$ test or one-way analysis of variance (ANOVA) was performed to analyze statistical differences between groups by using GraphPad Prism 5 (GraphPad Inc., La Jolla, CA, USA). $P<0.05$ was regarded as statistically significant.

\section{Results}

miR-203 is lowly expressed and associated with radiosensitivity in GC

To investigate the role of miR-203 in regulating radiosensitivity of GC, its expression was first measured in GC. Compared with NT group, the expression of miR203 was significantly decreased in GC tissues (Figure 1A). Similarly, the abundance of miR-203 was abnormally reduced in MKN-45, SGC-7901 and AGS cells compared with that in GES-1 cells (Figure 1B). Moreover, MKN-45 cells with lower expression of miR-203 were relatively more resistant to radiation, and AGS cells showed relative more radiosensitivity (Figure 1C). Hence, the MKN-45 and AGS cells were used for further experiments.

\section{miR-203 enhances radiosensitivity of GC} cells in vitro and in vivo

To explore the effect of miR-203 on radiosensitivity, MKN-45 cells were transfected with miR-203 or miR-NC and AGS cells were transfected with anti-miR-203 or antimiR-NC. As a result, the abundance of miR-203 was effectively elevated in miR-203-transfected MKN-45 cells and obviously reduced in anti-miR-203-transfected AGS cells (Figure 2A and B). The results of colony formation revealed that overexpression of miR-203 significantly inhibited survival fraction in MKN-45 cells, while its knockdown increased the survival fraction in AGS cells after different doses of radiation treatment (Figure $2 \mathrm{C}$ and D). However, miR-203 overexpression showed little effect on radiosensitivity of GES-1 cells except at 8 Gy (Figure S1). Moreover, MTT assay demonstrated that addition of miR-203 led to great reduction of cell viability in MKN-45 cells after different doses of radiation treatment (Figure 2E). However, inhibition of miR-203 caused an opposite effect on cell viability in AGS (Figure 2F). Besides, as shown in Figure $2 \mathrm{G}$ and $2 \mathrm{H}$, up-regulation of miR-203 promoted cell apoptosis in radiation-treated MKN-45 cells, but miR-203 down-regulation significantly impeded cell apoptosis in AGS cells after treatment of radiation. Additionally, the radiosensitive role of miR-203 was also analyzed in mice by xenograft and treatment of radiation. In MKN-45 cells-formed xenograft model, overexpression of miR-203 resulted in obvious loss of tumor volume and weight after treatment of radiation compared with miR-NC group (Figure $3 \mathrm{~A}$ and $\mathrm{B}$ ). However, after exposure of radiation, anti-miR-203 group showed higher tumor volume and weight than anti-miR-NC group in AGS-induced xenograft model (Figure 3C and D).

\section{ZEBI is a target of miR-203}

To explore the mechanism allows miR-203 participating in GC, the potential target of miR-203 was probed by TargetScan Release 7.2. As shown in Figure 4A, bioinformatics analysis showed the putative binding sites of miR-203
A

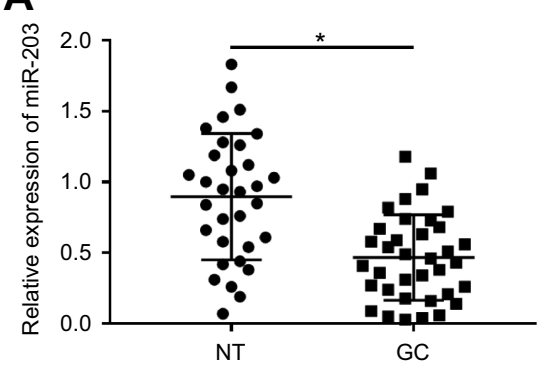

B

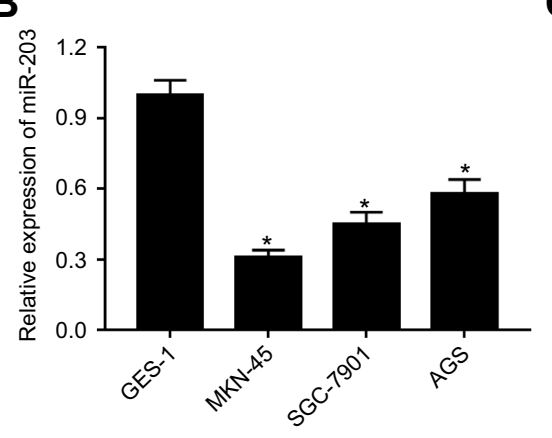

C

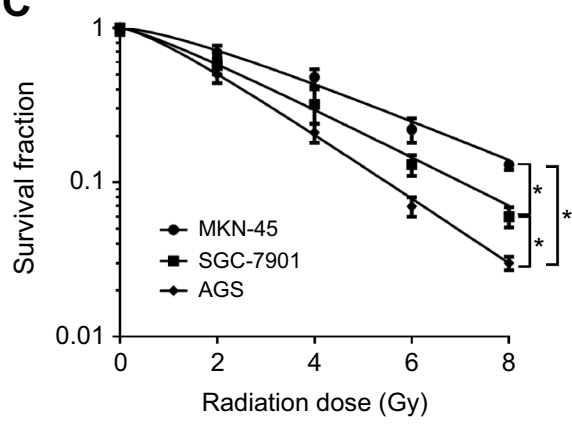

Figure I miR-203 expression is down-regulated in GC. (A) The expression of miR-203 was measured in GC tissues and NT samples by quantitative real-time polymerase chain reaction. (B) The level of miR-203 was detected in GC cells by quantitative real-time polymerase chain reaction. (C) Survival fraction was analyzed in GC cells by colony formation assay. $* P<0.05$. 
A

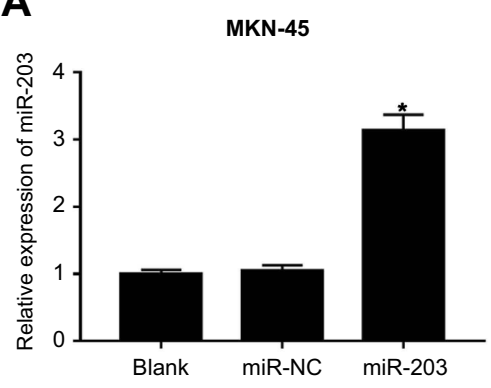

D

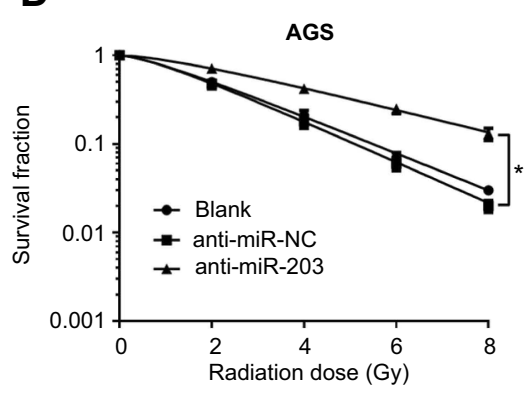

B

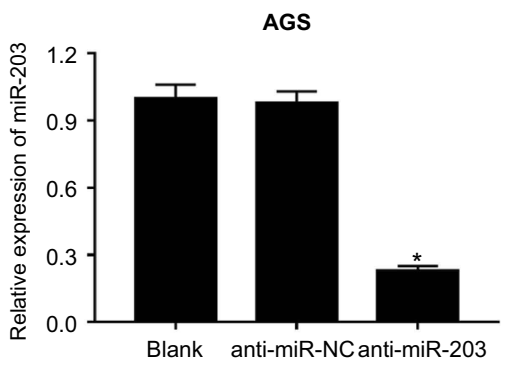

E
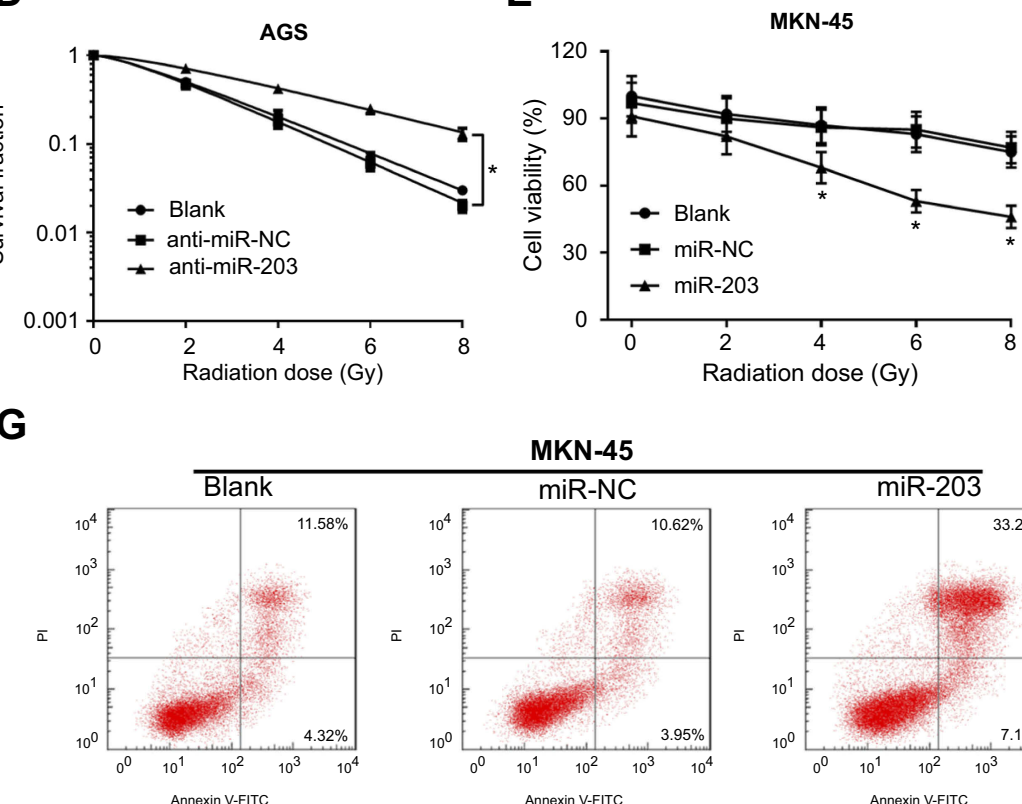

H

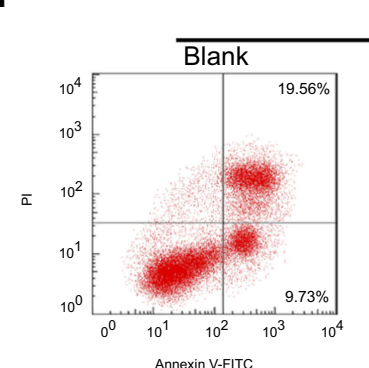

MKN-45

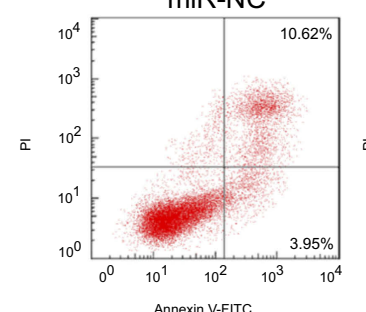

Annexin V-FITC

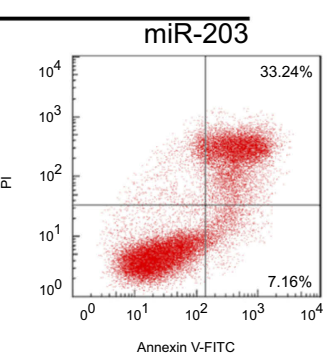

Annexin V-FITC
AGS
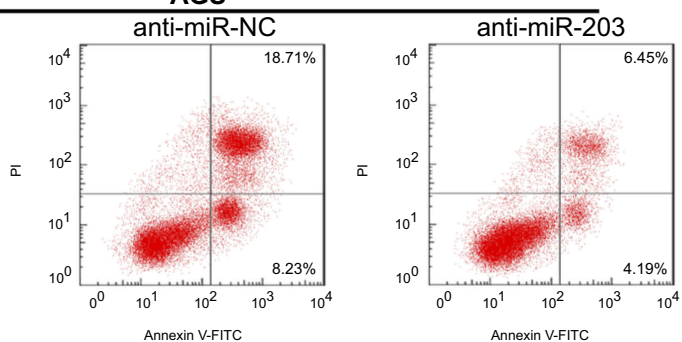

C

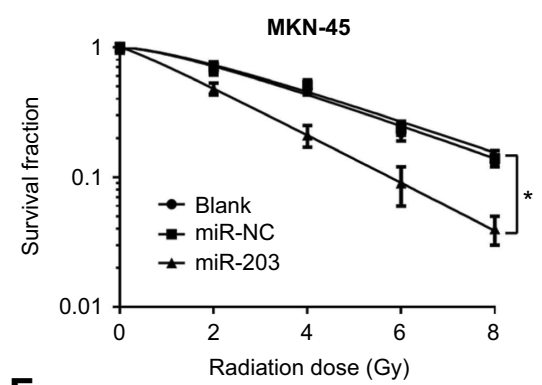

$\mathbf{F}$

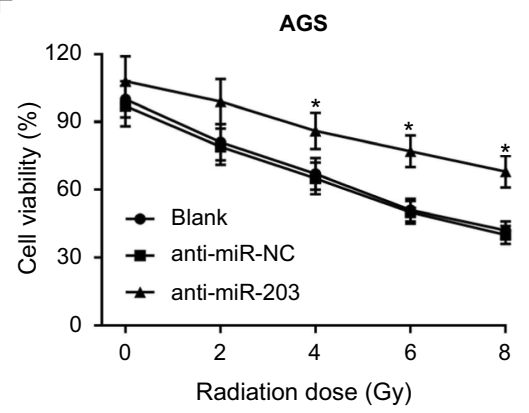

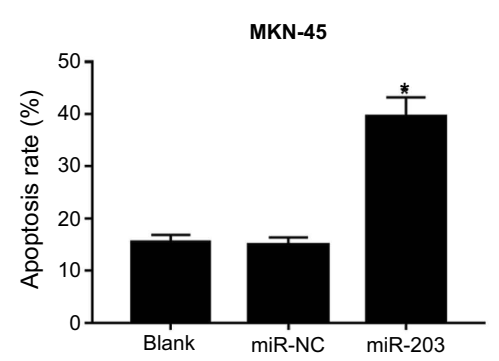

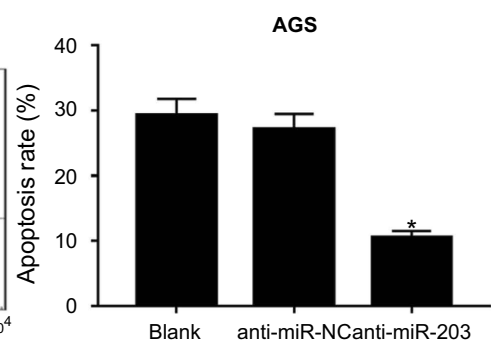

Figure 2 miR-203 enhances radiosensitivity in GC cells. (A and B) The abundance of miR-203 was measured in MKN-45 cells transfected with miR-203 or miR-NC and AGS cells transfected with anti-miR-203 or anti-miR-NC by quantitative real-time polymerase chain reaction. Survival fraction (C and D), cell viability (E and $\mathbf{F}$ ) and apoptosis $(\mathbf{G}$ and $\mathbf{H})$ were measured in MKN-45 cells transfected with miR-203 or miR-NC and AGS cells transfected with anti-miR-203 or anti-miR-NC after treatment of radiation by colony formation, MTT or flow cytometry, respectively. $* P<0.05$.

and ZEB1. This prediction was identified in MKN-45 and AGS cells by luciferase activity assay. Results showed that accumulation of miR-203 led to a great loss of luciferase activity in MKN-45 cells transfected with ZEB1-Wt, while it did not affect the activity in empty vector group (Figure 4B). However, abrogation of miR-203 significantly increased the luciferase activity in AGS cells transfected with ZEB1-Wt, whereas its efficacy was lost in response to empty vector group (Figure 4C). Moreover, overexpression of miR-203 notably suppressed ZEB1 expression at protein and mRNA levels in MKN-45 cells, but its knockdown evidently enhanced ZEB1 abundance in AGS cells (Figure 4D-4G). Subsequently, the expression of ZEB1 was measured in GC tissues and results showed that ZEB1 mRNA expression was abnormally elevated in GC tissues compared with that in NT samples (Figure 4H). Besides, the abundance of ZEB1 mRNA in GC tissues was negatively correlated with miR203 level $(\mathrm{r}=-0.462, P=0.005)$ (Figure 4I). 
A

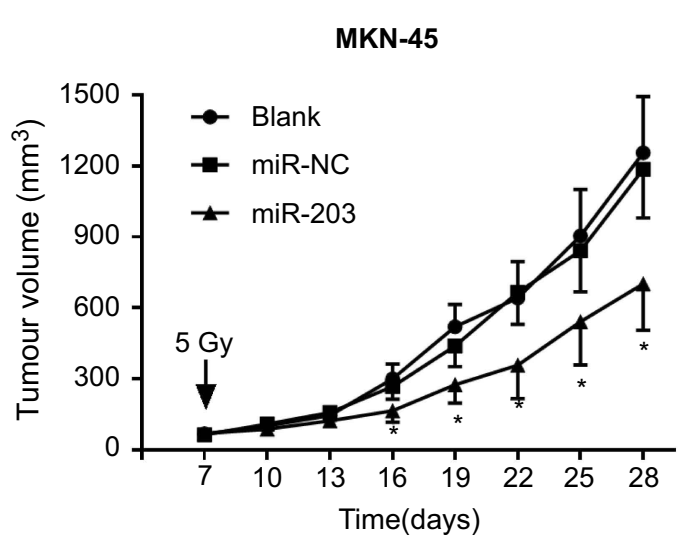

C

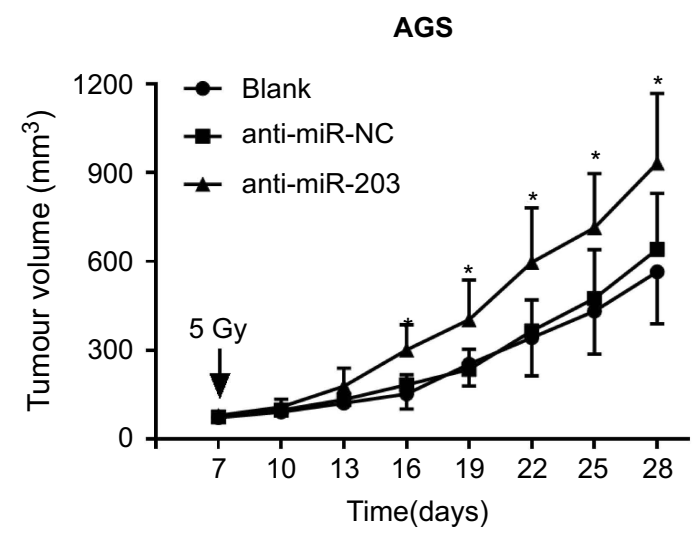

B

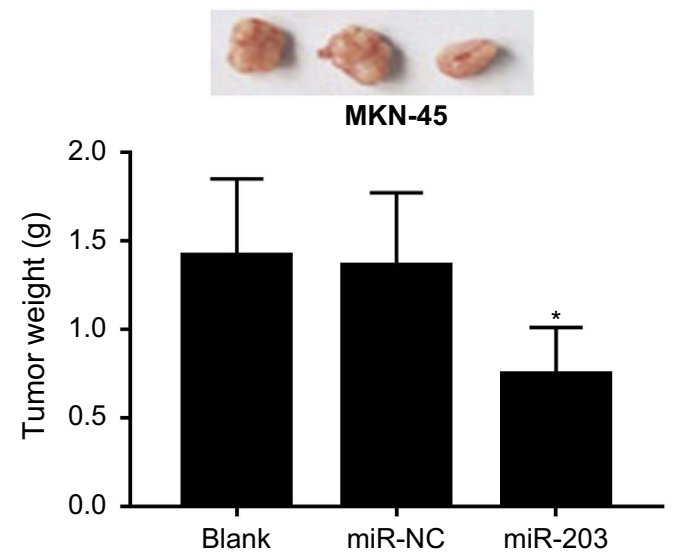

D

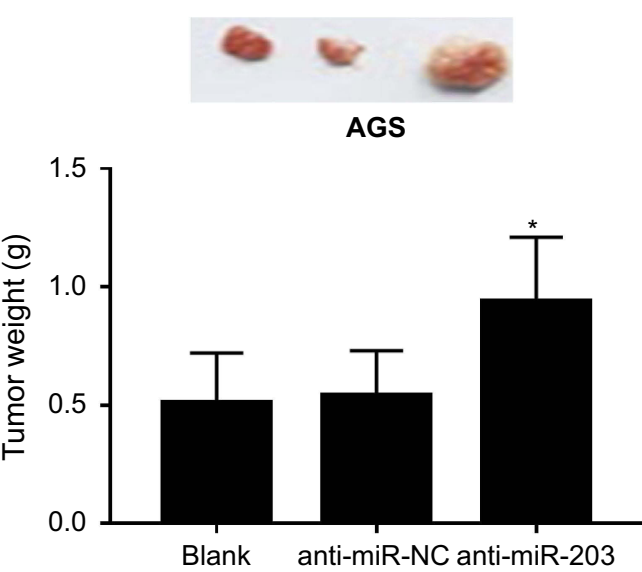

Figure 3 miR-203 promotes tumor inhibitory ability of radiation in mice. (A and B) The tumor volume and weight were measured in xenograft model formed by MKN-45 stably transfected with miR-203 or miR-NC after treatment of radiation. (C and D) The tumor volume and weight were detected in xenograft model formed by AGS stably transfected with anti-miR-203 or anti-miR-NC after treatment of radiation. $* P<0.05$.

\section{ZEBI is associated with miR-203-} mediated radiosensitivity of GC cells

As a result, the abundance of ZEB1 protein was effectively enhanced in MKN-45 cells transfected with ZEB1 and was evidently reduced in AGS cells transfected with shZEB1 compared with that in their corresponding controls (Figure $5 \mathrm{~A}$ and $\mathrm{B})$. To investigate whether ZEB1 was required for miR-203-mediated regulation of radiosensitivity, MKN-45 cells were co-transfected with miR-203 and ZEB1 or vector and AGS cell were co-transfected with anti-miR-203 and shZEB1 or scrambled. As displayed in Figure 5C, 5E and $5 \mathrm{G}$, restoration of ZEB1 significantly increased the survival fraction and cell viability but decreased apoptosis in MKN-45 cells with miR-203 overexpression after radiation treatment. However, the opposite occurred in response to ZEB1 interference in AGS cells with miR-203 deficiency after treatment of radiation (Figure $5 \mathrm{D}, 5 \mathrm{~F}$ and $5 \mathrm{H})$. Moreover, the role of ZEB1 was further evaluated in vivo. The mice were injected with MKN-45 cells stably transfected with miR-203 and ZEB1 or vector or AGS cells stably transfected with anti-miR-203 and shZEB1 or scrambled. Results showed that tumor volume and weight were significantly elevated in miR-203+ZEB1 group compared with those in ZEB1+vector group in MKN-45induced xenograft model after exposure of radiation (Figure 6A and B). However, the tumor growth showed an opposite trend in anti-miR-203+shZEB1 group in radiation-treated AGS xenograft model (Figure 6C and D).

\section{Discussion}

In recent years, great advances have been gained in epidemiology, prevention and therapy of GC. ${ }^{23}$ However, aberrant molecular signatures related to resistance drive GC malignancy. ${ }^{24}$ The former findings have suggested that miRNAs play essential roles in diagnosis, prognosis and chemo- and radiotherapy in different cancers, including 
A

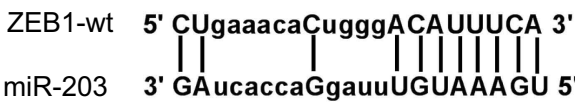

D
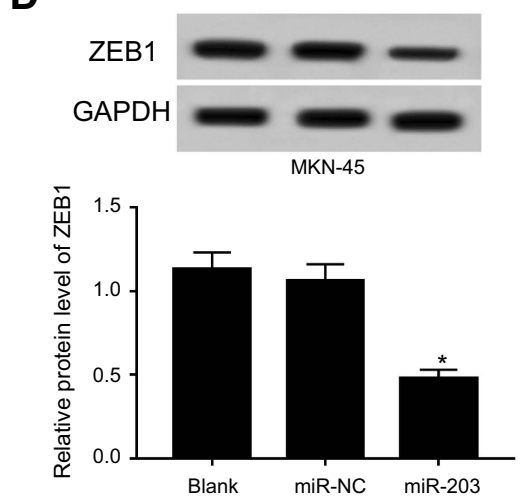

G

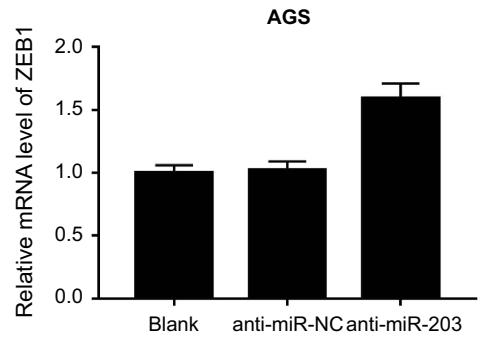

B

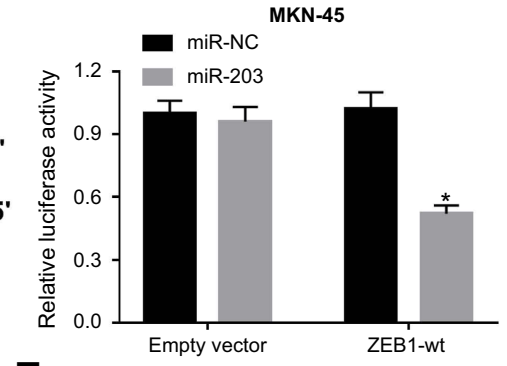

E
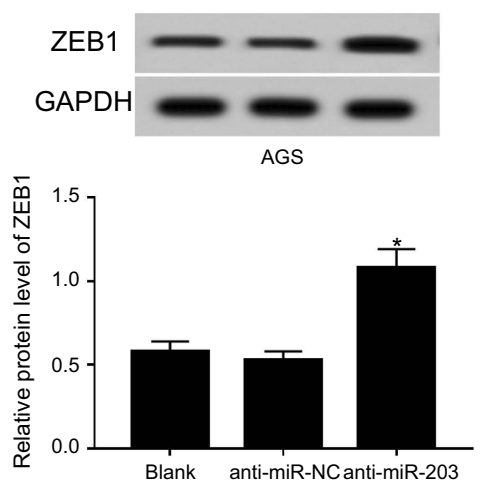

H

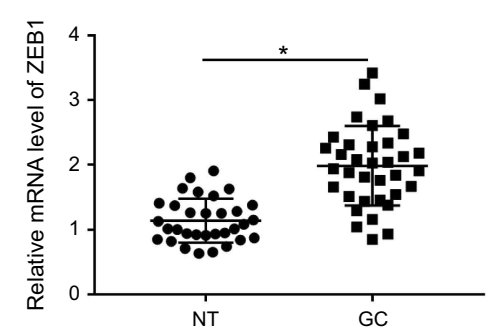

C

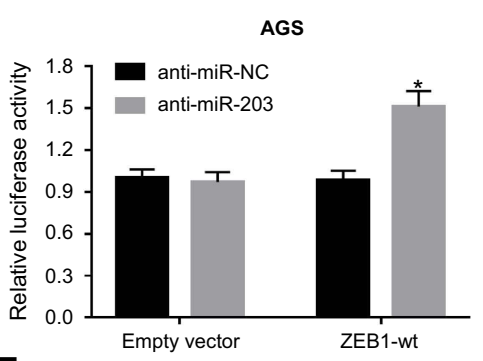

F

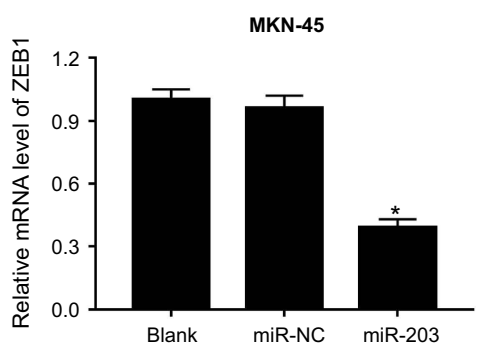

I

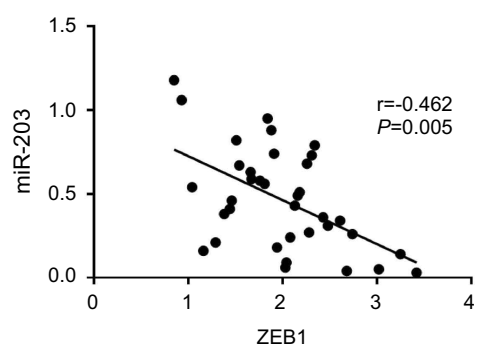

Figure 4 ZEBI is a target of miR-203. (A) The potential binding sites of miR-203 and ZEBI was predicted by TargetScan Release 7.2. (B and C) Luciferase activity was measured in MKN-45 cells transfected with empty vector or ZEBI-Wt and miR-203 or miR-NC and AGS cells transfected with empty vector or ZEBI-Wt and anti-miR-203 or anti-miR-NC. (D-G) The expression of ZEBI was measured at protein and mRNA levels in MKN-45 cells transfected with miR-203 or miR-NC and AGS cells transfected with anti-miR-203 or anti-miR-NC by western blot or quantitative real-time polymerase chain reaction. (H) The expression of ZEBI mRNA was detected in GC tissues and NT samples by quantitative real-time polymerase chain reaction. (I) The relationship between miR-203 and ZEBI levels in GC tissues was explored by Spearman rank correlation. ${ }^{*} P<0.05$.

GC. $^{25,26}$ Here we found that miR-203 expression was down-regulated in GC tissues and cells, which is also in agreement with the previous study. ${ }^{27}$ Moreover, we found that miR-203 expression was associated with radiosensitivity of GC cells. In this study, we first investigated the sensibilization role of miR-203 in radiotherapy of GC cells and revealed the regulatory network of miR-203/ZEB1.

The available evidence has indicated that miR-203 reduced radioresistance by regulating interleukin 8 (IL-8) and AKT signaling in nasopharyngeal carcinoma. ${ }^{28}$ Moreover, Shao et al revealed that miR-203 promoted cell radiosensitivity in vitro and in vivo by targeting B-cell-specific moloney leukemia virus insertion site 1 (Bmi-1) in hepatocellular carcinoma. ${ }^{29}$ These findings indicated that
miR-203 might function as a radiosensitizer in human cancers. However, there is no work on the interaction between miR-203 and radiosensitivity of GC. In the present study, we disclosed that miR-203 promoted radiosensitivity of GC cells in vitro and in vivo, indicating the therapeutic value of miR203 in radiotherapy of GC. Nevertheless, the potential mechanism allows miR-203 addressing radiosensitivity of GC remain poorly understood. Functional miRNA is known to regulate target expression by binding its 3'-UTR sequences. Previous studies have reported multiple targets of miR-203 in various cancers, such as slug (SNAI2), regulator of $\mathrm{G}$ protein signaling 17 (RGS17), ataxia telangiectasia mutated kinase (ATM) and progesterone-induced blocking factor 1 (PIBF1). ${ }^{14,15,30,31}$ In this study, we validated ZEB1 
A
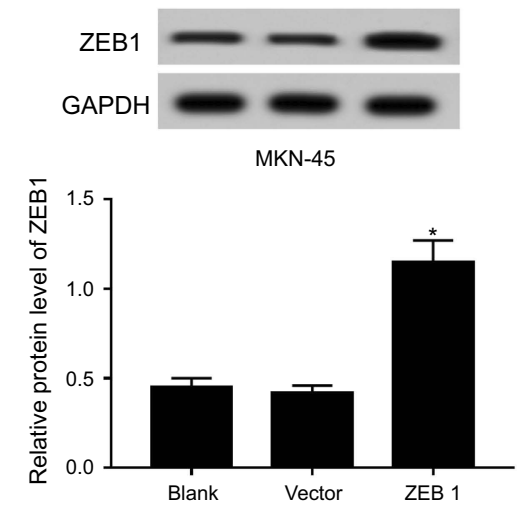

C

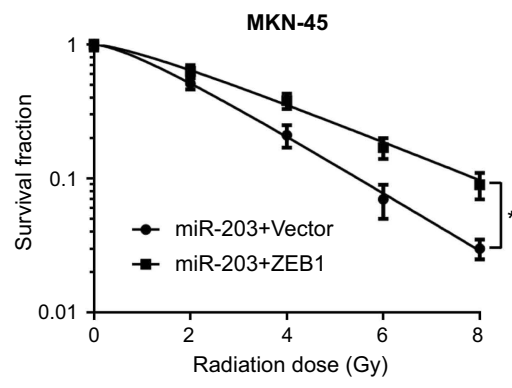

E

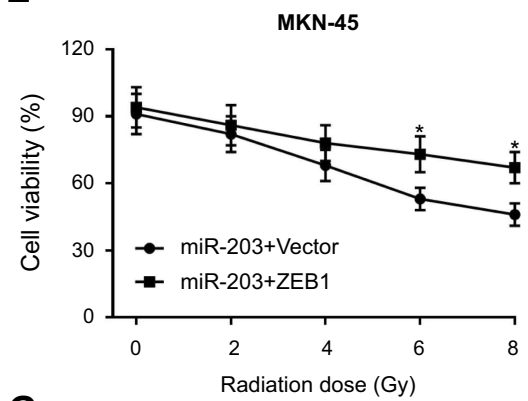

G
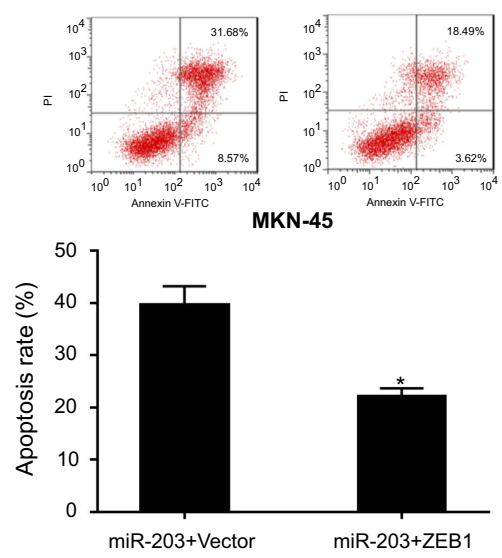

B
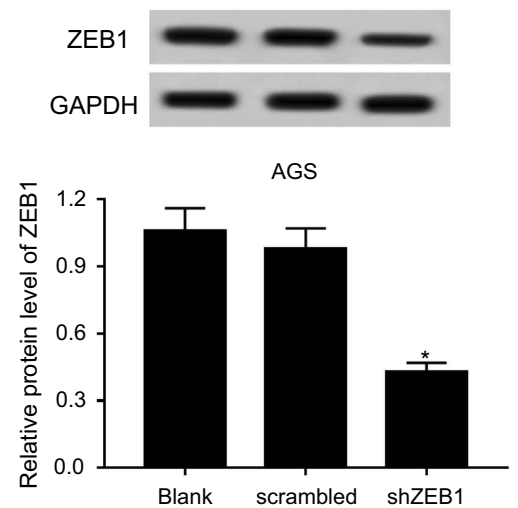

D

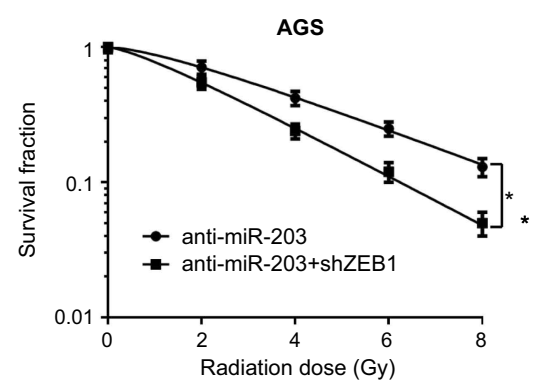

F

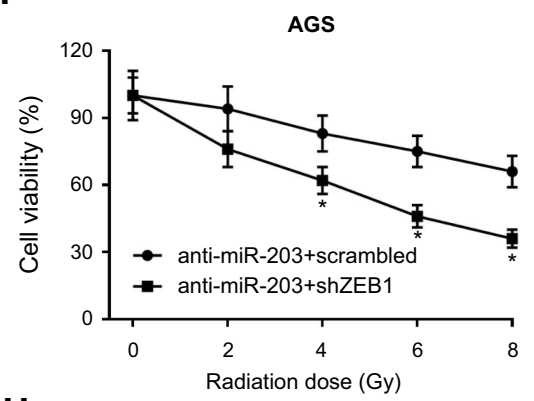

H

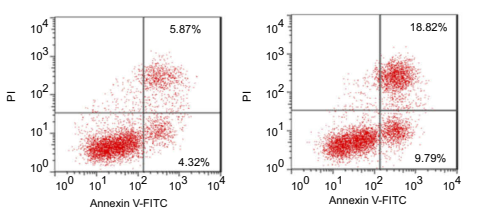

AGS

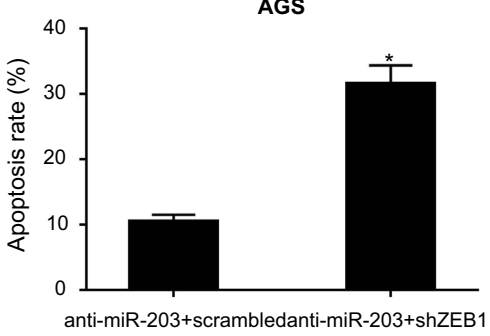

Figure 5 miR-203 regulates radiosensitivity in GC cells by targeting ZEBI. (A and B) The expression of ZEBI protein was measured in MKN-45 cells transfected with ZEBI or vector and AGS transfected with shZEBI or scrambled by western blot. Survival fraction (C and $\mathbf{D})$, cell viability (E and $\mathbf{F})$ and apoptosis (G and $\mathbf{H})$ were measured in MKN-45 cells transfected with miR-203+vector or miR-203+ZEBI and AGS cells transfected with anti-miR-203+scrambled or anti-miR-203+shZEBI after treatment of radiation by colony formation, MTT or flow cytometry, respectively. $* P<0.05$. 
A

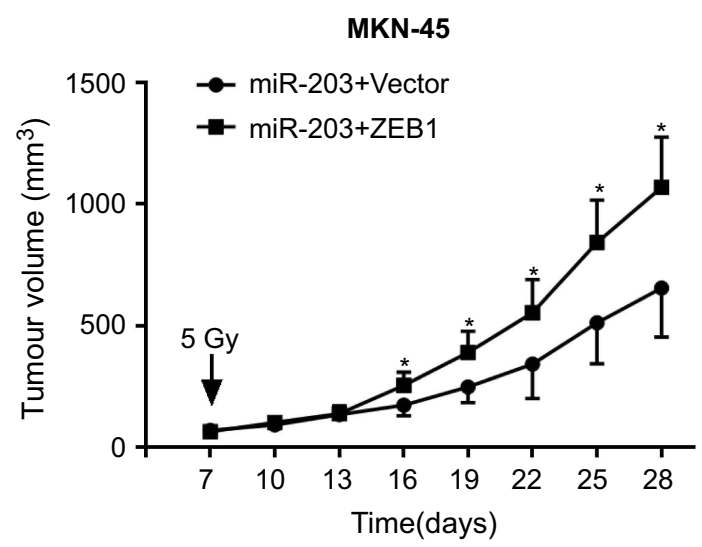

C

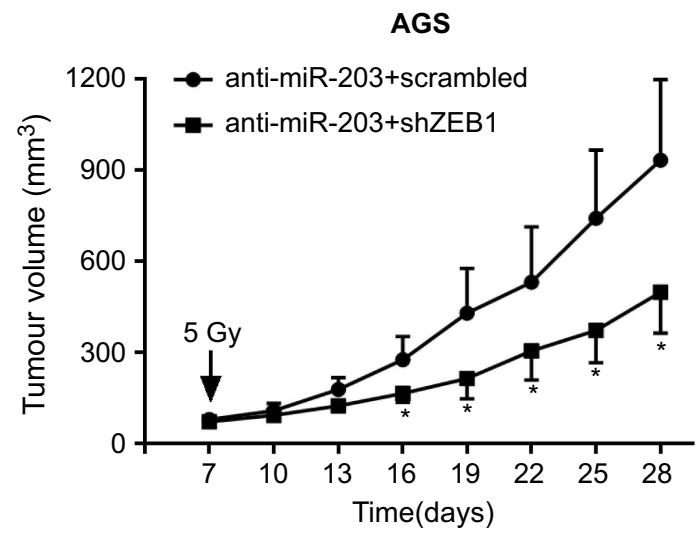

B

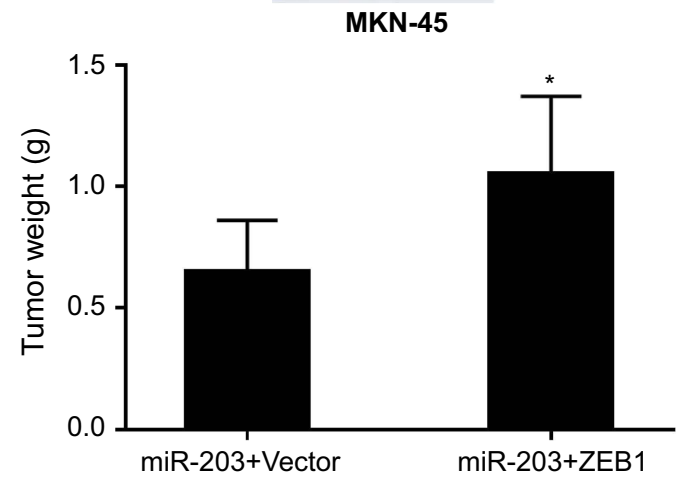

D

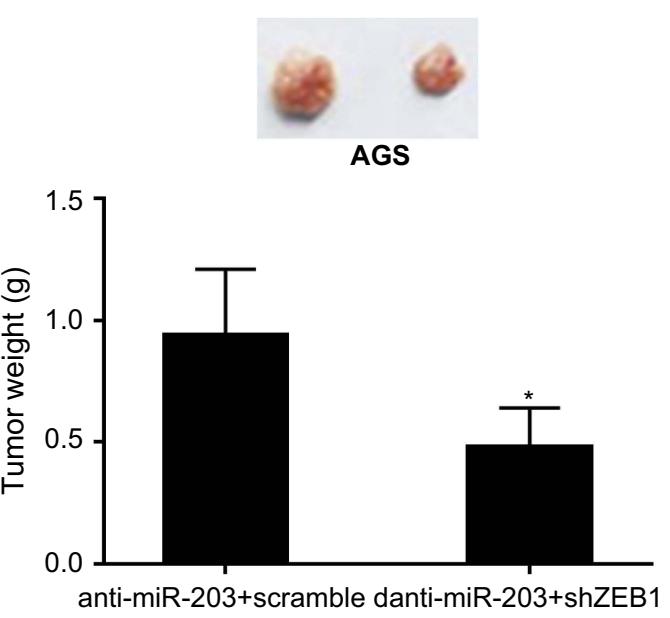

Figure 6 ZEBI is involved in the regulatory role of miR-203 in radiation-treated mice. (A and $\mathbf{B})$ The tumor volume and weight were measured in xenograft model formed by MKN-45 stably transfected with miR-203+vector or miR-203+ZEBI after treatment of radiation. (C and $\mathbf{D})$ The tumor volume and weight were detected in xenograft model formed by AGS stably transfected with anti-miR-203+scarmbled or anti-miR-203+shZEBI after treatment of radiation. $* P<0.05$.

as a target of miR-203 in GC cells by bioinformatics analysis and luciferase activity assay, which is also reported in nonsmall cell lung cancer. ${ }^{19}$

This study showed high expression of ZEB1 mRNA in GC tissues, which is also in agreement with previous studies. ${ }^{32}$ Furthermore, available evidences have indicated that ZEB1 is involved in proliferation, migration, invasion and epithelial-to-mesenchymal transition in GC progression. ${ }^{33,34}$ These findings suggested that ZEB1 could serve as an oncogene in GC progression. Notably, emerging evidence suggested that miR-205 promoted tumor radiosensitivity by targeting ZEB1 in breast cancer, which indicated the inhibitory effect of ZEB1 on radiosensitivity. ${ }^{35}$ In addition, sever such reports uncovered that inhibition of ZEB1 might protect radiosensitivity in glioblastoma, rectal cancer and prostate cancer. ${ }^{36-38}$ According to these reports, we hypothesized ZEB1 might also be involved in radioresistance of GC cells. Intriguingly, this research confirmed that ZEB1 impaired radiosensitivity, revealed by it reversed miR-203mediated promotion of radiosensitivity in GC, which suggested that miR-203 enhanced radiosensitivity of GC cells by targeting ZEB1 in vitro and in vivo. Previous effort has reported the importance of signaling pathway in radiosensitivity of cancers, such as PI3K/AKT/mTOR pathway and mitogen-activated protein kinase (MAPK)/extracellular signal-regulated kinase (ERK) pathway. ${ }^{39}$ Hence, the potential signaling pathway addressed by miR-203 is expected to be explored in further study.

\section{Conclusion}

In short, miR-203 expression was reduced and ZEB1 mRNA was enhanced in GC. Addition of miR-203 increased radiosensitivity of GC cells in vitro and in vivo, possibly by targeting ZEB1. This study 
indicates miR-203 as a promising radiosensitizer for GC treatment.

\section{Disclosure}

This work was supported by the Guizhou Provincial Science and Technology Foundation LS [2012](027). All the authors declare that they have no further financial or non-financial conflicts of interest in this research.

\section{References}

1. Van Cutsem E, Sagaert X, Topal B, Haustermans K, Prenen H. Gastric cancer. Lancet. 2016;388(10060):2654-2664. doi:10.1016/ S0140-6736(16)30354-3

2. Thrumurthy SG, Chaudry MA, Hochhauser D, Mughal M. The diagnosis and management of gastric cancer. Bmj. 2013;347:f6367. doi:10.1136/bmj.f6367

3. Hajj C, Goodman K. Role of radiotherapy and newer techniques in the treatment of GI cancers. J Clin Oncol. 2015;33(16):1737-1744. doi:10.1200/JCO.2014.59.9787

4. Zhang N, Fei Q, Gu J, Yin L, He X. Progress of preoperative and postoperative radiotherapy in gastric cancer. World J Surg Oncol. 2018;16(1):187. doi:10.1186/s12957-018-1490-7

5. Li H, Jin X, Chen B, Li P, Li Q. Autophagy-regulating microRNAs: potential targets for improving radiotherapy. $J$ Cancer Res Clin Oncol. 2018;144(9):1623-1634. doi:10.1007/s00432-018-2675-8

6. Yuan H, Wang T, Zhang K. MicroRNAs as potential biomarkers for diagnosis, therapy and prognosis of gastric cancer. Onco Targets Ther. 2018;11:3891-3900. doi:10.2147/OTT.S156921

7. He J, Hua J, Ding N, et al. Modulation of microRNAs by ionizing radiation in human gastric cancer. Oncol Rep. 2014;32(2):787-793. doi:10.3892/or.2014.3246

8. Shen Y, Bae I, Park G, Choi H, Lee K, Kim S. MicroRNA-196b enhances the radiosensitivity of SNU-638 gastric cancer cells by targeting RAD23B. Biomed Pharmacother. 2018;105:362-369. doi:10.1016/j.biopha.2018.05.111

9. Wang B, Li X, Zhao G, et al. miR-203 inhibits ovarian tumor metastasis by targeting BIRC5 and attenuating the TGF $\beta$ pathway. $J$ Exp Clin Cancer Res. 2018;37(1):235. doi:10.1186/s13046-0180906-0

10. Ji D, Jiang C, Zhang L, et al. LncRNA CRNDE promotes hepatocellular carcinoma cell proliferation, invasion, and migration through regulating miR-203/BCAT1 axis. J Cell Physiol. 2019;234(5):65486560. doi: $10.1002 /$ jcp. 27396

11. Chen L, Ding Z, Zhang Y, He S, Wang X. MiR-203 over-expression promotes prostate cancer cell apoptosis and reduces ADM resistance. Eur Rev Med Pharmacol Sci. 2018;22(12):3734-3741.

12. Chang J, Hwang Y, Lee D, et al. MicroRNA-203 modulates the radiation sensitivity of human malignant glioma cells. Int J Radiat Oncol Biol Phys. 2016;94(2):412-420. doi:10.1016/j.ijrobp.2015.10.001

13. Gao P, Wang S, Jing F, Zhan J, Wang Y. microRNA-203 suppresses invasion of gastric cancer cells by targeting ERK1/2/Slug/E-cadherin signaling. Cancer Biomark. 2017;19(1):11-20. doi:10.3233/CBM160167

14. Zhou P, Jiang N, Zhang G, Sun Q. MiR-203 inhibits tumor invasion and metastasis in gastric cancer by ATM. Acta Biochim Biophys Sin. 2016;48(8):696-703. doi:10.1093/abbs/gmw063

15. Chu S, Wang G, Zhang P, et al. MicroRNA-203 suppresses gastric cancer growth by targeting PIBF1/Akt signaling. J Exp Clin Cancer Res. 2016;35:47. doi:10.1186/s13046-016-0444-6

16. Caramel J, Ligier M, Puisieux A. Pleiotropic Roles for ZEB1 in Cancer. Cancer Res. 2018;78(1):30-35. doi:10.1158/0008-5472. CAN-17-2476
17. Zhang P, Sun Y, Ma L. ZEB1: at the crossroads of epithelialmesenchymal transition, metastasis and therapy resistance. Cell Cycle. 2015;14(4):481-487. doi:10.1080/15384101.2015.1006 048

18. Yuan G, Quan J, Dong D, Wang Q. Long noncoding RNA CAT104 promotes cell viability, migration, and invasion in gastric carcinoma cells through activation of microrna-381-inhibiting zinc finger E-box-binding homeobox 1 (ZEB1) expression. Oncol Res. 2018;26(7):1037-1046. doi:10.3727/096504017X151447484 28127

19. Singh T, Prasad R, Katiyar S. Therapeutic intervention of silymarin on the migration of non-small cell lung cancer cells is associated with the axis of multiple molecular targets including class 1 HDACs, ZEB1 expression, and restoration of miR-203 and E-cadherin expression. Am J Cancer Res. 2016;6(6):1287-1301.

20. Livak KJ, Schmittgen TD. Analysis of relative gene expression data using real-time quantitative PCR and the $2-\Delta \Delta \mathrm{CT}$ method. Methods. 2001;25(4):402-408. doi:10.1006/meth.2001.1262

21. Cui FB, Liu Q, Li RT, et al. Enhancement of radiotherapy efficacy by miR-200c-loaded gelatinase-stimuli PEG-Pep-PCL nanoparticles in gastric cancer cells. Int $J$ Nanomedicine. 2014;9:2345-2358. doi:10.2147/IJN.S60874

22. Hu C, Gao X, Han Y, et al. Evodiamine sensitizes BGC-823 gastric cancer cells to radiotherapy in vitro and in vivo. Mol Med Rep. 2016;14:413-419. doi:10.3892/mmr.2016.5237

23. Venerito M, Vasapolli R, Rokkas T, Malfertheiner P. Gastric cancer: epidemiology, prevention, and therapy. Helicobacter. 2018;23(suppl 1):e12518.

24. Duarte H, Gomes J, Machado J, Reis C. Gastric cancer: basic aspects. Helicobacter. 2018;23(suppl 1):e12523.

25. Da Silva Oliveira K, Thomaz Araújo T, Albuquerque C, et al. Role of miRNAs and their potential to be useful as diagnostic and prognostic biomarkers in gastric cancer. World $J$ Gastroenterol. 2016;22 (35):7951-7962. doi:10.3748/wjg.v22.i37.8314

26. Hummel R, Hussey D, Haier J. MicroRNAs: predictors and modifiers of chemo- and radiotherapy in different tumour types. Eur J Cancer. 2010;46(2):298-311. doi:10.1016/j.ejca.2009.10.027

27. Zheng Y, Liu W, Guo L, Yang X. The expression level of miR-203 in patients with gastric cancer and its clinical significance. Pathol Res Pract. 2017;213(12):1515-1518. doi:10.1016/j.prp.2017.09.025

28. Qu J, Yi H, Ye X, et al. MiRNA-203 reduces nasopharyngeal carcinoma radioresistance by targeting IL8/AKT signaling. Mol Cancer Ther. 2015;14(11):2653-2664. doi:10.1158/1535-7163. MCT-15-0461

29. Shao Y, Zhang D, Li X, et al. MicroRNA-203 increases cell radiosensitivity via directly targeting Bmi-1 in hepatocellular carcinoma. Mol Pharm. 2018;15(8):3205-3215. doi:10.1021/acs.molpharmaceut. $8 \mathrm{~b} 00302$

30. Tian X, Tao F, Zhang B, Dong J, Zhang Z. The miR-203/SNAI2 axis regulates prostate tumor growth, migration, angiogenesis and stemness potentially by modulating GSK-3 $\beta / \beta$-CATENIN signal pathway. IUBMB Life. 2018;70(3):224-236. doi:10.1002/iub.1928

31. Chi Y, Jin Q, Liu X, et al. miR-203 inhibits cell proliferation, invasion, and migration of non-small-cell lung cancer by downregulating RGS17. Cancer Sci. 2017;108(12):2366-2372. doi:10.1111/cas.13401

32. Zhou X, Wang Y, Shan B, et al. The downregulation of miR-200c/ 141 promotes ZEB1/2 expression and gastric cancer progression. Med Oncol. 2015;32(1):428. doi:10.1007/s12032-014-0428-3

33. Song Z, Li W, Wang L, Jia N, Chen B. MicroRNA-454 inhibits tumor cell proliferation, migration and invasion by downregulating zinc finger E-box-binding homeobox 1 in gastric cancer. Mol Med Rep. 2017;16(6):9067-9073. doi:10.3892/mmr.2017.7758

34. Xu C, Li M, Zhang L, et al. MicroRNA-205 suppresses the invasion and epithelial-mesenchymal transition of human gastric cancer cells. Mol Med Rep. 2016;13(6):4767-4773. doi:10.3892/ mmr.2016.5118 
35. Zhang P, Wang L, Rodriguez-Aguayo C, et al. miR-205 acts as a tumour radiosensitizer by targeting ZEB1 and Ubc13. Nat Commun. 2014;5:5671. doi:10.1038/ncomms5972

36. Kowalski-Chauvel A, Modesto A, Gouaze-Andersson V, et al. Alpha6 integrin promotes radioresistance of glioblastoma by modulating DNA damage response and the transcription factor Zeb1. Cell Death Dis. 2018;9(9):872. doi:10.1038/s41419-018-1111-y

37. Shao M, Bi T, Ding W, et al. OCT4 potentiates radio-resistance and migration activity of rectal cancer cells by improving epithelialmesenchymal transition in a ZEB1 dependent manner. Biomed Res Int. 2018;2018:3424956. doi:10.1155/2018/3424956
38. El Bezawy R, Cominetti D, Fenderico N, et al. miR-875-5p counteracts epithelial-to-mesenchymal transition and enhances radiation response in prostate cancer through repression of the EGFR-ZEB1 axis. Cancer Lett. 2017;395:53-62. doi:10.1016/j.canlet.2017.02.033

39. Ni J, Bucci J, Chang L, Malouf D, Graham P, Li Y. Targeting MicroRNAs in prostate cancer radiotherapy. Theranostics. 2017;7 (13):3243-3259. doi:10.7150/thno.19934 


\section{Supplementary material}

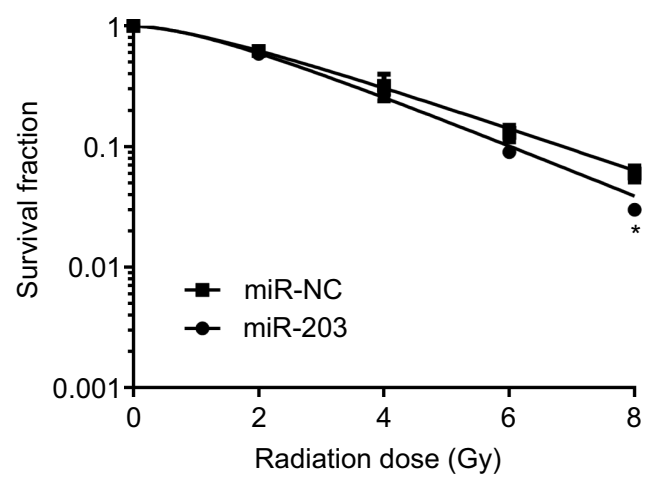

Figure SI The effect of miR-203 on survival fraction of GES-I cells after radiation treatment. GES-I cells were transfected with miR-203 mimic or miR-NC and then exposed to different doses $(0,2,4,6$ and $8 \mathrm{~Gy})$ of radiation. The survival fraction of cells was analyzed by colony formation assay. $* P<0.05$.

\section{Publish your work in this journal}

OncoTargets and Therapy is an international, peer-reviewed, open access journal focusing on the pathological basis of all cancers, potential targets for therapy and treatment protocols employed to improve the management of cancer patients. The journal also focuses on the impact of management programs and new therapeutic

Submit your manuscript here: https://www.dovepress.com/oncotargets-and-therapy-journal agents and protocols on patient perspectives such as quality of life, adherence and satisfaction. The manuscript management system is completely online and includes a very quick and fair peer-review system, which is all easy to use. Visit http://www.dovepress.com/ testimonials.php to read real quotes from published authors. 